\title{
Concurrent chronic neutrophilic leukemia blast crisis and multiple myeloma: A case report and literature review
}

\author{
JINNING SHI ${ }^{1}$, YING NI ${ }^{2}$, JIANYONG LI $^{2}$, HAIRONG QIU ${ }^{2}$ and KOURONG MIAO ${ }^{2}$ \\ ${ }^{1}$ Department of Hematology, The Affiliated Jiangning Hospital, Nanjing Medical University; \\ ${ }^{2}$ Department of Hematology, The First Affiliated Hospital of Nanjing Medical University, \\ Jiangsu Hospital, Nanjing, Jiangsu 210029, P.R. China
}

Received March 22, 2014; Accepted January 8, 2015

DOI: $10.3892 / 01.2015 .3043$

\begin{abstract}
The current study presents the case of a 78-year-old male with concurrent chronic neutrophilic leukemia (CNL) and multiple myeloma (MM) who developed acute myeloid leukemia after two years of treatment with hydroxyurea, cyclophosphamide, prednisone and thalidomide. The patient presented with mature neutrophilic leukocytosis, hepatosplenomegaly, a high neutrophil alkaline phosphatase score and an absence of the Philadelphia chromosome or the BCR-ABL fusion gene. A bone marrow aspirate smear and biopsy indicated that the CNL coexisted with a plasma cell neoplasm. In addition, monoclonal $\lambda$-paraproteinemia was detected by serum protein immunofixation electrophoresis, and bone lesions were identified in multiple vertebrae. The patient achieved complete remission following one cycle of induction chemotherapy with the decitabine regimen in combination with the low-dose cytarabine, aclarubicin and granulocyte-colony stimulating factor (CAG) priming regimen. The occurrence of CNL and MM concurrently is extremely rare and thus, it has only been reported in a small number of cases. The occurrence of CNL and MM in the same patient as two distinct hematological malignancies indicates the neoplastic transformation of a pluripotent stem cell. Decitabine combined with the CAG priming regimen may present a good therapeutic strategy for elderly patients with secondary acute myeloid leukemia.
\end{abstract}

\section{Introduction}

Chronic neutrophilic leukemia (CNL) presents as a distinct clinicopathological entity, exhibiting the characteristics of mature neutrophilic leukocytosis and splenomegaly with bone marrow granulocytic hyperplasia without evidence of dysplasia

Correspondence to: Dr Kourong Miao, Department of Hematology, The First Affiliated Hospital of Nanjing Medical University, Jiangsu Hospital, 300 Guangzhou Road, Nanjing, Jiangsu 210029, P.R. China

E-mail:kourongmiao@163.com

Key words: chronic neutrophilic leukemia, multiple myeloma, acute monocytic leukemia or striking reticulin fibrosis (1). Multiple myeloma (MM) is a condition that is associated with the monoclonal proliferation of plasma cells that have differentiated from lymphoid B cells (2). Thus, the abnormal cell types observed in CNL and $\mathrm{MM}$ are markedly different, originating from myeloid and lymphoplasmacytic cell lineages, respectively $(3,4)$. A small number of cases of CNL occurring concurrently with MM have been reported, however, the clinicopathological characteristics of the disease remain unclear, and the clonal cytogenetic abnormalities have not been identified in this specific group of patients. The current study presents the case of a patient with concurrent CNL and MM who subsequently developed acute myeloid leukemia. The patient achieved complete remission (CR) following induction chemotherapy with decitabine and the cytarabine, aclarubicin and granulocyte-colony stimulating factor (G-CSF) (CAG) priming regimen. Consent was obtained from the family of the patient.

\section{Case report}

A 78-year-old male presented with leukocytosis (leukocyte count, 47,500/ $\mu \mathrm{l}$; normal range, 4,000-10,000/ $\mu \mathrm{l}$ ) on a routine complete blood count prior to cataract surgery in The First Affiliated Hospital of Nanjing Medical University, Jiangsu Hospital, (Nanjing, China) in 2009. Hemoglobin and thrombocyte counts were within the normal ranges. The patient experienced no subjective symptoms. Moderate hepatosplenomegaly was identified, however, no lymphadenopathy was evident. A bone marrow aspirate smear revealed hypercellularity with a large number of neutrophils, including segmented neutrophils (40.4\%), band forms (19.6\%), myelocytes (13.6\%), lymphocytes $(6 \%)$ and a few rare cells, such as blast cells and plasma cells.. The peripheral blood film contained segmented neutrophils $(80 \%)$, band forms $(6 \%)$, lymphocytes $(11 \%)$, monocytes $(2 \%)$ and eosinophils $(1 \%)$.

Neutrophil alkaline phosphatase staining indicated that the neutrophils were strongly positive with a high score. Conventional cytogenetic analysis was performed on bone marrow cells using the direct method (5), as well as $24 \mathrm{~h}$ of non-stimulated culture of fresh heparinized bone marrow cells. R-banding results revealed that the karyotype of the patient was 46,XY [20]. Dual color dual fusion fluorescence in situ hybridization (FISH) using BCR/ABL probes (Vysis, Inc., Downers 


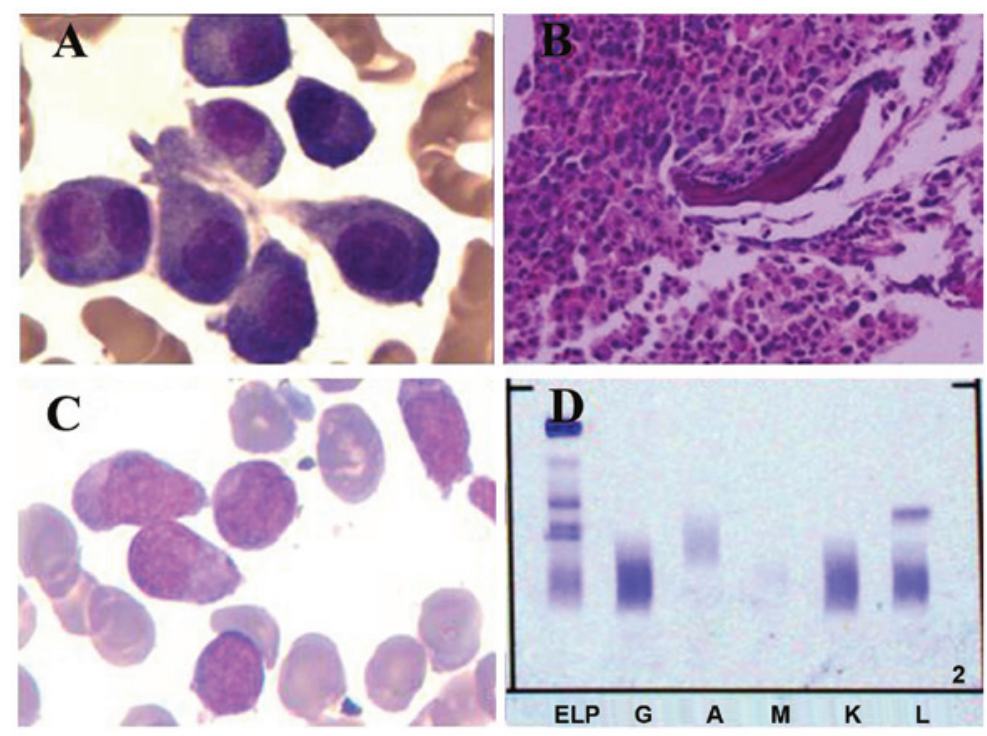

Figure 1. A bone marrow aspirate and biopsy were performed. (A) Bone marrow aspirate results showing a cluster of abnormal plasma cells, including multinucleated plasma cells. (B) Bone marrow biopsy analysis showing an accumulation of myeloid cells, dominated by band forms and segmented neutrophils. A number of clusters of middle-sized cells with abundant cytoplasm were identified and immunochemistry results were CD38(+), CD138(+) and epithelial membrane antigen(+/-). (C) Bone marrow smear results demonstrating hypercellularity, with monoblast cells and promonocytes accounting for 58\% of nucleated cells, indicating that the patient had progressed to acute monocytic leukemia. (D) Serum protein immunofixation electrophoresis showing a sharp positive band for the $\lambda$-light chain subtype. CD, cluster of differentiation. ELP, electrophoresis of protein; G, IgG; A, IgA; M, IgM; K, $\kappa$ light chain; L, $\lambda$ light chain.

Grove, IL, USA) to detect the Philadelphia chromosome was also performed to exclude chronic myeloid leukemia (CML), and the results showed that screening for BCR/ABL fusion was negative in all 300 examined interphase cells. Polymerase chain reaction analysis was performed to determine $\mathrm{BCR} / \mathrm{ABL}$ fusion gene status, which was also confirmed to be negative. Reactive neutrophilia and other myeloproliferative neoplasms were excluded by clinical and laboratory observation. A diagnosis of CNL was established according to the criteria of the World Health Organization Classification of Tumors of Hematopoietic and Lymphoid Tissues (6) and thus, the patient was administered hydroxyurea at a dose of $1 \mathrm{~g}$ twice per day., with the dose adjusted according to the leukocyte count.

After 14 months, a bone marrow aspirate revealed that the number of plasma cells was increased to $6.4 \%$, including plasmablastic, immature plasma and multinucleated plasma cells (Fig. 1). The bone marrow biopsy indicated the coexistence of myeloproliferative and plasma neoplasms. The abnormal plasma cells were cluster of differentiation (CD)38(+), CD138(+) and epithelial membrane antigen(+/-) (Fig. 1). Karyotype analysis was repeated and again revealed a karyotype of 46,XY [15]. Interphase FISH analysis combined with magnetic-activated cell sorting (MACS), which markedly enhances the sensitivity of cytogenetic analysis, was performed. Five different probes specific for the regions containing $13 q 14.3,14 q 32,1 q 21,1 p 12$ and $17 \mathrm{p} 13$ were used to detect chromosomal aberrations. Positive values were identified for $13 q 14.3,17 \mathrm{p} 13$ and 1q21, while negative values were identified for the probes specific for $1 \mathrm{p} 13$ and $14 q 32$. Immunoglobulin (Ig) tests showed an IgM level of $0.27 \mathrm{~g} / 1$ (normal range, 0.4-2.3 g/l), while IgG and IgA levels were within the normal ranges. The $\beta 2$ microglobulin level was $5.17 \mathrm{mg} / 1$ (normal range, $0-3 \mathrm{mg} / \mathrm{l}$ ) and the urine $\kappa$ - and $\lambda$-light chains for $24 \mathrm{~h}$ were 70.8 and $434 \mathrm{mg}$, respectively. Serum protein immunofixation electrophoresis showed a sharp positive band for the $\lambda$-light chain subtype (Fig. 1). Bone magnetic resonance imaging (MRI) indicated diffused lesions in multiple vertebrae. The concurrent existence of MM was established and thus, cyclophosphamide (50mg daily, d1-21), prednisone (30mg daily, d1-21) and thalidomide (100mg, every night) were subsequently administered for the treatment of MM. Each cycle was 1 month.

After two years, the patient's bone marrow smear demonstrated hypercellularity, with monoblast cells and promonocytes accounting for $58 \%$ of nucleated cells, indicating that the patient had progressed to acute monocytic leukemia, according to the French-American-British classification criteria (Fig. 1) (7). The patient achieved CR after one cycle of induction chemotherapy with the decitabine regimen $\left(15 \mathrm{mg} / \mathrm{m}^{2}\right.$ every day, on days 1-5) combined with low-dose CAG (10 mg/m² cytarabine, every $12 \mathrm{~h}$, on days 3-9; $10 \mathrm{mg}$ aclarubicin, on days 3-6; and $200 \mu \mathrm{g} / \mathrm{m}^{2} \mathrm{G}-\mathrm{CSF}$, every day). Each cycle was 1 month. Subsequent consolidation chemotherapy (cytarabine $2 \mathrm{~g} / \mathrm{m}^{2}$ twice daily for 3 days) was administered. The patient completed two cycles in our hospital and refused any other chemotherapy. After approximately one year, the patient relapsed and succumbed to the disease soon after.

\section{Discussion}

$\mathrm{CNL}$ is a rare myeloproliferative neoplasm that is characterized by persistent leukocytosis consisting of mature neutrophils, bone marrow granulocytic hyperplasia, hepatosplenomegaly and the absence of the Philadelphia chromosome or BCR-ABL fusion gene. The incidence and pathogenesis of CNL remains unclear. In $\leq 20 \%$ of reported cases, neutrophilia is associated with an underlying neoplasm, most frequently MM (6). The coexistence of CNL and MM is extremely rare and thus, only a small number of cases have been reported $(3,4)$. However, MM coexisting with other myeloproliferative neoplasms, including CML (8), polycythemia vera (9), essential thrombocytosis (10) 
and primary myelofibrosis (11), has been reported, which may indicate an association between these distinct hematological disorders, rather than a random simultaneous occurrence.

In total, $\sim 90 \%$ of CNL patients do not exhibit any cytogenetic abnormalities. In the remaining patients, aberrant clonal karyotypes may include $\operatorname{add}(8), \operatorname{add}(9), \operatorname{add}(21), \operatorname{del}(20 q)$, $\operatorname{del}(11 q)$ and del(12p) (3). In the present case, no clonal chromosome abnormalities were identified by the conventional cytogenetic method. However, clonal abnormalities, such as del(13q14.3), del(17p13) and add(1q21), were detected by the FISH technique using MACS to purify MM cells, which confirmed it as malignant disease.. Standen et al (3) reported two cases of CNL and concurrent MM, and identified a disproportionate excess of $\lambda$-light chain restriction in those patients. The study also reported that IgG- $\lambda$-type paraprotein and $\lambda$-light chains were identified in four and nine of the 11 previously reported cases of CNL associated with MM, respectively (4). In CML patients with concurrent MM, IgG-א-type monoclonal paraprotein was predominant in the reported cases (9). In the present case, the patient was confirmed as $\lambda$-type by immunofixation electrophoresis, identical to the main type ( $\lambda$-type) of CNL concurrent with MM. The patient received chemotherapy for MM and disease progression did not occur for 2 years.

CNL is generally considered as a slowly progressive disorder (3); however, the survival of patients is variable, ranging between six months and $>20$ years. In the present study, the patient developed acute monocytic leukemia after three years, which indicated a poor prognosis. The differential diagnosis of chronic myelomonocytic leukemia was re-evaluated and excluded on the basis of a low monocyte count. The patient developed MM after the diagnosis of CNL, and succumbed to blast crisis within two years. Whether MM accelerates this process remains unclear and thus, the mechanism of this specific disorder requires further investigation.

The patient presented in this case was 78 years old and the performance status revealed that intensive chemotherapy was unsuitable. Hypermethylation, which is an epigenetic mutation that leads to a decrease in transcription via promoter regions of tumor suppressor genes causing gene silencing, has been identified as an important promoter of myeloid leukemogenesis (12). Therefore, demethylating agents are being investigated to reverse the silencing of critical tumor suppressor genes. Decitabine is a demethylating agent that functions as a cytosine analogue. The CAG regimen, which was originally designed in Japan, rapidly became popularized in China for the treatment of acute myeloid leukemia and myelodysplastic syndrome patients due to the relatively mild toxicity exhibited by the regimen. The $\mathrm{CR}$ rate of acute myeloid leukemia patients treated with the CAG regimen (56.7\%) appears to be marginally lower than the CR rate of patients treated with the standard daunorubicin and cytarabine regimen (DA; $45 \mathrm{mg} / \mathrm{m}^{2}$ daunorubicin and $100-200 \mathrm{mg} / \mathrm{m}^{2}$ cytarabine; $57-65 \%$ ) (13). However, no randomized control trial studies regarding the efficacy of the CAG and DA regimens have been performed. In the present study, the patient achieved CR following combined treatment with decitabine and the CAG regimen, which exhibited a low toxicity when compared with standard chemotherapy.

In this case, a CNL patient with concurrent MM, which was confirmed by bone marrow examination, immunofixation electrophoresis and MRI techniques, was presented. We hypothesize that the neutrophilia was a neoplasm or secondary to abnormal cytokine release from the neoplastic plasma cells or other cells regulated by plasma cells. In this case, CNL was diagnosed prior to MM, and then it transformed into acute monocytic leukemia, indicating that the neutrophilia was malignant and a diagnosis of CNL concurrent with MM was accepted. The occurrence of the two malignancies in one patient supports the hypothesis that the two malignancies are derived from hematopoietic progenitor stem cells, which may differentiate into lymphoplasmacytic or myeloid cell lines. Treatment with decitabine in combination with the CAG priming regimen may present a good treatment strategy for elderly patients with acute myeloid leukemia.

\section{Acknowledgements}

This study was supported by the National Natural Science Foundation of China (grant no. 81000216).

\section{References}

1. Elliott MA: Chronic neutrophilic leukemia: a contemporary review. Curr Hematol Rep 3: 210-217, 2004.

2. Bird JM, Owen RG, D'Sa S, et al; Haemato-oncology Task Force of British Committee for Standards in Haematology (BCSH) and UK Myeloma Forum: Guidelines for the diagnosis and management of multiple myeloma 2011. Br J Haematol 154: $32-75,2011$

3. Standen GR, Jasani B, Wagstaff M and Wardrop CA: Chronic neutrophilic leukemia and multiple myeloma. An association with lambda light chain expression. Cancer 66: 162-166, 1990.

4. Dinçol G, Nalçaci M, Doğan O, Aktan M, Küçükkaya R, Ağan M and Dinçol K: Coexistence of chronic neutrophilic leukemia with multiple myeloma. Leuk Lymphoma 43: 649-651, 2002.

5. Shen Y, Xue Y, Li J, Pan J and Wu Y: Clinical, cytogenetic and dual-color FISH studies on five cases of myelodysplastic syndrome or acute myeloid leukemia patients with 1;7 translocation. Chin Med J (Engl). 116:231-234, 2003.

6. Swerdlow SH, Campo E, Harris NL, Jaffe ES, Pileri SA, Stein $\mathrm{H}$, et al (eds): WHO Classification of Tumours of Haematopoietic and Lymphoid Tissues. 4th edition. IARC Press, Lyon, France, 2008.

7. Bennett JM, Catovsky D, Daniel MT, Flandrin G, Galton DA, Gralnick HR and Sultan C: Proposals for the classification of the acute leukaemias. French-American-British (FAB) co-operative group. Br J Haematol. 33:451-458,1976.

8. Tanaka M, Kimura R, Matsutani A, Zaitsu K, Oka Y and Oizumi K: Coexistence of chronic myelogenous leukemia and multiple myeloma. Case report and review of the literature. Acta Haematol 99: 221-223, 1998.

9. Chang H and Shih LY: Concurrence of multiple myeloma and idiopathic erythrocytosis. Acta Clin Belg 64: 434-435, 2009.

10. Eskazan AE, Ongoren S, Ar MC, Soysal T, Ferhanoglu B, Aki H and Aydin Y: Essential thrombocythemia and multiple myeloma: two rare diseases in one patient. Clin Lymphoma Myeloma Leuk 11: 442-445, 2011

11. Kawauchi K, Mori H, Sugiyama H, Oshimi K and Hirayama A: Multiple myeloma with coexistent myelofibrosis: improvement of myelofibrosis following recovery from multiple myeloma after treatment with melphalan and prednisolone. Jpn J Med 30: 483-486, 1991.

12. Ganetsky A: The role of decitabine for the treatment of acute myeloid leukemia. Ann Pharmacother. 46:1511-1517,2012.

13. Wei G, Ni W, Chiao JW, Cai Z, Huang H and Liu D: A meta-analysis of CAG (cytarabine, aclarubicin, G-CSF) regimen for the treatment of 1029 patients with acute myeloid leukemia and myelodysplastic syndrome. J Hematol Oncol 4: 46, 2011. 